\title{
Market Efficiency of Ruble-RMB Exchange Rates: Long-run Equilibrium, VAR Estimates and Granger Causality Tests
}

\author{
Gaolu ZOU \\ Faculty of Tourism and Industrial Development, Chengdu University, Chengdu, China
}

\begin{abstract}
China-Russia Ruble-RMB currency swap agreement may have long-run and short-run exchange risks for China. This paper mainly aims to investigate the informational efficiency of Ruble-RMB foreign exchange market. Methods used include the ADF and PP tests, the Perron test, the Johansen trace and Engle-Granger tests, vector autoregressive model and Granger causality test. Cointegration does not exist between the exchange rates of Ruble, dollar, euro, yen and pound to RMB, suggesting long-run informationally efficient. We constructed a first-differenced VAR for these exchange rates. We find a unidirectional Granger causality from euro-RMB exchange rate to Ruble-RMB exchange rate, suggesting short-run informationally inefficient. Hence, the Ruble-RMB foreign exchange market violates the efficient market hypothesis $(\mathrm{EMH})$ and the Ruble-RMB currency swaps may not receive diversification benefits in the short run.
\end{abstract}

KEYWORD: Cointegration; exchange rate; efficient market hypothesis; Granger causality; structural break; vector autoregressive model

\section{INTRODUCTION}

China and Russia signed an agreement for RubleRMB currency swaps in mid-October 2014, with a total value of RMB 150 billion/RUB 815 billion. The agreement is for three years with a choice for the eventual extension. Ruble devalued by $32.5 \%$ from 20 October 2014-20 December, compared to RMB (China Foreign Exchange 2014). Hence, a high exchange rate uncertainty would exist if China has signed the swap agreement. However, we must obtain empirical evidence for the argument. Exchange rates of RMB against the world main currencies including US dollar, euro, Japanese yen and pound may have a long-run equilibrium.

Cointegration and Granger causality suggest the long-run equilibrium and the short-run dynamics, respectively (Engle \& Granger 1987, Granger 1980). Cointegration and Granger causality violate the efficient market hypothesis (EMH) (Granger 1981). $\mathrm{EMH}$ implies that investors would achieve diversification benefits (Fama 1970). Therefore, the paper aims to investigate the information efficiency of Ruble-RMB foreign exchange market. Empirical evidence is implicative for examination of RubleRMB currency swap risks.

\section{METHODS AND DATA}

The Johansen trace method and Engle-Granger technique are used to test for long-run equilibrium represented by cointegration (Engle \& Granger 1987, Johansen 1991). We test for unit roots using the ADF and PP tests (Dickey \& Fuller 1979, Phillips \& Perron 1988). We test for a structural break using the Perron test (Model C) (Perron 1997). Cointegration suggests presence of an errorcorrection model (ECM):

$$
\Delta y_{t}=\lambda+\sum_{k=1}^{m} \pi_{k} \Delta y_{t-k}+\sum_{k=1}^{m} \xi_{k} \Delta x_{t-k}+\delta z_{t-1}+\varepsilon_{t}
$$

Where $y_{\mathrm{t}}, x_{\mathrm{t}}$ are $I(1)$ time-series variables. $z_{\mathrm{t}-1}$ is the error-correction (EC) term representing a long-run equilibrium. However, removing the EC term from an ECM, a short-run vector autoregression model (VAR) is still valid where there is no cointegration (Engle \& Granger 1987). In addition, Granger causality (Granger 1969) from $x_{\mathrm{t}}$ to $y_{\mathrm{t}}$ exists if all

$$
\xi_{k}=0
$$

Where $\xi_{k}$ 's are the coefficients of lagged $\boldsymbol{x}_{\mathrm{t}}$ in the ECM. Wald- $\chi^{2}$ statistics are used to drive the Granger causality test. 
Exchange rates comprise those of Ruble to RMB $(R U B)$ and US dollar to RMB (DOLLAR). They also include those of the euro to RMB (EURO), Japanese yen to RMB (YEN), and pound to RMB (POUND). Excluding weekends and holidays, data has 963 daily observations from 4 January 2011 to December 24, 2014 (China Foreign Exchange 2014).

Table 1. Statistics for the exchange rate of one hundred foreign currency to RMB.

\begin{tabular}{|c|c|c|c|c|c|}
\hline Variable & DOLLAR & EURO & YEN & POUND & RUB \\
\hline Mean & 627.80 & 837.99 & 7.05 & 1004.89 & 19.55 \\
\hline Median & 628.60 & 829.65 & 7.41 & 1002.85 & 19.84 \\
\hline Maximum & 663.49 & 964.45 & 8.37 & 1085.18 & 23.77 \\
\hline Minimum & 609.30 & 748.48 & 5.05 & 915.62 & 8.82 \\
\hline $\begin{array}{c}\text { Standard } \\
\text { Deviation }\end{array}$ & 13.45 & 45.00 & 1.01 & 34.74 & 2.44 \\
\hline Skewness & 0.72 & 0.80 & $(0.17)$ & $(0.02)$ & $(0.98)$ \\
\hline Kurtosis & 2.70 & 3.14 & 1.38 & 2.37 & 5.02 \\
\hline $\begin{array}{c}\text { Jarque-Bera } \\
p \text {-value }\end{array}$ & $\begin{array}{c}85.75 \\
0.00\end{array}$ & $\begin{array}{c}102.56 \\
0.00\end{array}$ & $\begin{array}{c}109.63 \\
0.00\end{array}$ & $\begin{array}{c}16.03 \\
0.00\end{array}$ & $\begin{array}{c}318.42 \\
0.00\end{array}$ \\
\hline
\end{tabular}

\section{EMPIRICAL RESULTS}

The five time-series variables each contain a unit root (Table 2, Table 3). Both the trace and EngleGranger tests suggest no cointegration for these series (Table 4, Table 5). So we estimated a firstdifferenced VAR. EURO Granger caused $R U B$ at the $5 \%$ confidence level but not vice versa.

Table 2. Unit root tests.

\begin{tabular}{|c|c|c|c|c|c|c|}
\hline \multirow{2}{*}{$\begin{array}{l}\text { The log of } \\
\text { a variable }\end{array}$} & Level & $p$ & $k$ & $\begin{array}{c}\text { First } \\
\text { difference }\end{array}$ & $p$ & $k$ \\
\hline & \multicolumn{6}{|l|}{$\mathrm{ADF}$} \\
\hline DOLLAR & -2.56 & 0.30 & 3 & -16.19 & 0.00 & 3 \\
\hline EURO & -1.69 & 0.76 & 3 & -11.10 & 0.00 & 6 \\
\hline YEN & -1.83 & 0.69 & 3 & -9.10 & 0.00 & 9 \\
\hline POUND & -1.91 & 0.65 & 3 & -11.20 & 0.00 & 6 \\
\hline$R U B$ & 1.49 & 1.00 & 17 & -4.50 & 0.00 & 21 \\
\hline \multicolumn{7}{|c|}{ PP } \\
\hline DOLLAR & -2.21 & 0.48 & 15 & -29.87 & 0.00 & 15 \\
\hline EURO & -1.72 & 0.74 & 3 & -32.90 & 0.00 & 3 \\
\hline YEN & -1.66 & 0.77 & 3 & -29.90 & 0.00 & 3 \\
\hline POUND & -1.90 & 0.65 & 5 & -32.70 & 0.00 & 4 \\
\hline$R U B$ & 0.18 & 0.99 & 25 & -28.40 & 0.00 & 26 \\
\hline
\end{tabular}

Notes: $k$ denotes lag length. For the ADF tests, $k$ was chosen by the modified AIC. For the PP test, $k$ was selected by the Newey-West method (Newey \& West 1987). $k$ was selected between 3 and 25 following ( $\mathrm{Ng} \&$ Perron 1995). $p$ (-value) is in (MacKinnon 1996). Tests contained the trend and constant (Hamilton 1994, Hendry \& Juselius 2000).
Table 3. Perron structural break tests.

\begin{tabular}{|c|c|c|c|c|c|c|}
\hline $\begin{array}{c}\text { The log of } \\
\text { a variable }\end{array}$ & $\alpha$ & $\begin{array}{c}\text { Standard } \\
\text { Error }\end{array}$ & $t_{\alpha}^{*}$ & $p$ & Lag & $T_{\mathrm{b}}$ \\
\hline DOLLAR & 0.99 & 0.00 & 206.41 & 0.00 & 11 & 283 \\
\hline$E U R O$ & 0.98 & 0.01 & 152.46 & 0.00 & 8 & 411 \\
\hline$Y E N$ & 0.99 & 0.01 & 151.27 & 0.00 & 10 & 493 \\
\hline POUND & 0.99 & 0.01 & 161.90 & 0.00 & 8 & 631 \\
\hline$R U B$ & 0.97 & 0.01 & 100.45 & 0.00 & 10 & 897 \\
\hline
\end{tabular}

Estimates were for $\alpha$ on $y_{\mathrm{t}-1} . k$ is the lag order. Tests fixed the $k$ between 2 and 12 according to $(\mathrm{Ng}$ $\&$ Perron 1995). $t$-statistic represented that for the coefficient of the $k$ th lagged term. $t$-statistic exceeded or equaled to 1.8 in terms of absolute value according to (Perron 1989). The fraction $\lambda$ was 0.05 ; therefore, regressions were executed from $T=$ 48 to 915 ( $T$ is the sample size). $T_{\mathrm{b}}$ was the possible break point detected. The critical values for $T=100$ were $-6.07,-5.48$, and -5.17 at the $1 \%, 5 \%$, and $10 \%$ confidence levels, respectively (Table 1 , Panel $\mathrm{d}$ in (Perron 1997)).

Table 4. Engle-Granger cointegration tests.

\begin{tabular}{|l|c|c|}
\hline The log of the dependent variable & $\mathrm{Z}_{\alpha}$ & $p$-value* \\
\hline DOLLAR & -16.2 & 0.67 \\
\hline EURO & -12.20 & 0.85 \\
\hline YEN & -9.11 & 0.94 \\
\hline POUND & -12.9 & 0.82 \\
\hline RUB & -2.6 & 1.00 \\
\hline
\end{tabular}

$\mathrm{Z}_{\alpha}$ is the test statistic. Null hypothesis: the series were not cointegrated. Test equations contained the trend and constant. The lag lengths were chosen using modified AIC. * $p$-value in (MacKinnon 1996).

Table 5. Johansen multivariate cointegration trace tests.

\begin{tabular}{|c|c|c|c|c|c|c|c|}
\hline$r$ & Lag & Trace & $p^{*}$ & $\begin{array}{l}5 \% \\
\text { O-L }\end{array}$ & $\begin{array}{c}5 \% \\
\text { C\&L }\end{array}$ & JB & $\begin{array}{c}\text { Adj. Q- } \\
\text { statistic (lag, } \\
p \text {-value) }\end{array}$ \\
\hline 0 & \multirow[t]{3}{*}{4} & 69.7 & 0.52 & 88.8 & 90.5 & \multirow[t]{3}{*}{$\begin{array}{c}141568 \\
(0.00)\end{array}$} & \multirow[t]{3}{*}{$39(5,0.74)$} \\
\hline$\leq 1$ & & 44.4 & 0.68 & 63.9 & 65.1 & & \\
\hline$\leq 2$ & & 22.2 & 0.91 & 42.9 & 43.7 & & \\
\hline 0 & \multirow[t]{3}{*}{8} & 93.2 & 0.02 & 88.8 & 92.3 & \multirow[t]{3}{*}{$\begin{array}{l}86499 \\
(0.00)\end{array}$} & \multirow[t]{3}{*}{$47(9,0.43)$} \\
\hline$\leq 1$ & & 46.0 & 0.60 & 63.9 & 66.4 & & \\
\hline$\leq 2$ & & 27.5 & 0.65 & 42.9 & 44.6 & & \\
\hline 0 & \multirow[t]{3}{*}{14} & 79.4 & 0.20 & 88.8 & 95.1 & \multirow[t]{3}{*}{$\begin{array}{l}71512 \\
(0.00)\end{array}$} & \multirow[t]{3}{*}{$44(15,0.56)$} \\
\hline$\leq 1$ & & 44.6 & 0.67 & 63.9 & 68.4 & & \\
\hline$\leq 2$ & & 27.0 & 0.68 & 42.9 & 45.9 & & \\
\hline 0 & \multirow[t]{3}{*}{20} & 83.1 & 0.12 & 88.8 & 98.0 & \multirow[t]{3}{*}{$\begin{array}{l}47453 \\
(0.09)\end{array}$} & \multirow[t]{3}{*}{$70(21,0.01)$} \\
\hline$\leq 1$ & & 41.4 & 0.80 & 63.9 & 70.5 & & \\
\hline$\leq 2$ & & 24.4 & 0.82 & 42.9 & 47.4 & & \\
\hline
\end{tabular}


5\% O-L denotes 5\% asymptotical critical value (Osterwald-Lenum 1992). C\&L is $5 \%$ Cheung-Lai finite-sample critical value (Cheung \& Lai 1993). * $p$ (-value) in (MacKinnon, Haug \& Michelis 1999). JB and Adj. Q-statistic denotes multivariate JarqueBera normality statistic and Portmanteau autocorrelation test adjusted $Q$-statistic, respectively.

Table 6. VAR estimates and Granger causality tests.

\begin{tabular}{|c|c|c|c|c|}
\hline Variable in the log and first difference & Lagged term & Estimates & $t$-statistic & Granger causality $\left(\chi^{2}, p\right.$-value $)$ \\
\hline \multicolumn{5}{|l|}{ Dependent: $R U B$} \\
\hline \multirow[t]{10}{*}{$R U B$} & 1 & 0.03 & 0.88 & \\
\hline & 2 & 0.02 & 0.57 & \\
\hline & 3 & -0.03 & -0.77 & \\
\hline & 4 & -0.06 & -1.74 & \\
\hline & 5 & -0.15 & -3.66 & \\
\hline & 6 & -0.12 & -2.90 & \\
\hline & 7 & -0.03 & -0.56 & \\
\hline & 8 & 0.12 & 2.53 & \\
\hline & 9 & 0.15 & 3.18 & \\
\hline & 10 & 0.17 & 3.72 & \\
\hline \multirow[t]{10}{*}{ DOLLAR } & 1 & 0.33 & 0.63 & \multirow{10}{*}{$3.35,0.97$} \\
\hline & 2 & 0.00 & $\begin{array}{l}-0.01 \\
\end{array}$ & \\
\hline & 3 & -0.15 & -0.28 & \\
\hline & 4 & 0.19 & 0.35 & \\
\hline & 5 & 0.35 & 0.65 & \\
\hline & 6 & 0.09 & 0.17 & \\
\hline & 7 & 0.32 & 0.59 & \\
\hline & 8 & -0.64 & -1.20 & \\
\hline & 9 & 0.34 & 0.63 & \\
\hline & 10 & 0.27 & 0.53 & \\
\hline \multirow[t]{10}{*}{ EURO } & 1 & 0.13 & 1.52 & $20.4,0.03$ \\
\hline & 2 & -0.01 & -0.11 & \\
\hline & 3 & 0.08 & 0.96 & \\
\hline & 4 & 0.04 & 0.51 & \\
\hline & 5 & 0.15 & 1.68 & \\
\hline & 6 & 0.25 & 2.89 & \\
\hline & 7 & 0.08 & 0.86 & \\
\hline & 8 & -0.10 & -1.10 & \\
\hline & 9 & 0.10 & 1.13 & \\
\hline & 10 & -0.16 & -1.77 & \\
\hline \multirow[t]{10}{*}{ YEN } & 1 & 0.04 & 0.74 & \multirow[t]{10}{*}{$13,0.22$} \\
\hline & 2 & -0.02 & -0.38 & \\
\hline & 3 & 0.08 & 1.42 & \\
\hline & 4 & -0.07 & -1.23 & \\
\hline & 5 & -0.01 & -0.17 & \\
\hline & 6 & 0.06 & 1.02 & \\
\hline & 7 & 0.08 & 1.35 & \\
\hline & 8 & -0.01 & -0.11 & \\
\hline & 9 & 0.15 & 2.51 & \\
\hline & 10 & 0.00 & 0.02 & \\
\hline \multirow[t]{10}{*}{ POUND } & 1 & 0.05 & 0.58 & \multirow[t]{10}{*}{$6.54,0.76$} \\
\hline & 2 & -0.03 & -0.27 & \\
\hline & 3 & -0.03 & -0.37 & \\
\hline & 4 & -0.06 & -0.58 & \\
\hline & 5 & -0.01 & -0.07 & \\
\hline & 6 & -0.04 & -0.47 & \\
\hline & 7 & 0.08 & 0.81 & \\
\hline & 8 & -0.18 & -1.89 & \\
\hline & 9 & -0.10 & -1.08 & \\
\hline & 10 & -0.05 & -0.50 & \\
\hline Constant & & 0.00 & -1.14 & \\
\hline
\end{tabular}


Lags were chosen using AIC. $R$-squared $=0.11$. Adj. $R$-squared $=0.06 . \quad F$-statistic $=2.25 . \quad$ Akaike $\mathrm{AIC}=-6.34$. Schwarz $\mathrm{SC}=-6.08$. Autocorrelation LM statistic $=32(\mathrm{lag}=1, p$-value $=0.15)$.

\section{CONCLUDING REMARKS}

China has increased the number of its currency swap partners. Recent RMB-Ruble swap agreement suffers a considerable Ruble devaluation, showing existence of risks for the currency swaps. The risk arises from market efficiency across international exchange rates. Cointegration analysis does not suggest a long-run equilibrium between the exchange rates of the Ruble, dollar, euro, yen and pound to RMB. Despite this, we find Granger causality from euro-RMB exchange rate to RubleRMB exchange rate, which shows a violation of EMH. Therefore, Ruble-RMB swap risks exist for China at least in the short run.

\section{REFERENCES}

[1] Cheung, Y.-W. \& Lai, K.S. 1993. Finite-sample sizes of Johansen's likelihood ratio tests for cointegration. Oxford Bulletin of Economics and Statistics 55 (3): 313-328.

[2] China Foreign Exchange. 2014. Middle exchange rate of $R M B$. http://www.safe.gov.cn/wps/portal/english/Home.

[3] Dickey, D.A. \& Fuller, W.A. 1979. Distribution of the estimators for autoregressive time series with a unit root. Journal of the American Statistical Association 74 (386): 427-431.

[4] Engle, R.F. \& Granger, C.W.J. 1987. Cointegration and error correction: Representation, estimation and testing. Econometrica 55 (2): 251-276.

[5] Fama, E.F. 1970. Efficient Capital Markets: A Review of Theory and Empirical Work. Journal of Finance 25 (2): 383-417.
[6] Granger, C.W.J. 1969. Investigating causal relations by econometric models and cross-spectral methods. Econometrica 37 (3): 424-438.

[7] Granger, C.W.J. 1980. LONG MEMORY RELATIONSHIPS AND THE AGGREGATION OF DYNAMIC MODELS. Journal of Econometrics 14 (2): 227-238.

[8] Granger, C.W.J. 1981. Some properties of time series data and their use in econometric model specification. Journal of Econometrics 16 (1): 121-130.

[9] Hamilton, H.D. 1994. Time series analysis. Princeton, New Jersey: Princeton University Press.

[10] Hendry, D.F. \& Juselius, K. 2000. Explaining cointegration analysis: Part I. Energy Journal 21 (1): 142.

[11] Johansen, S. 1991. Estimation and hypotheses testing of co-integration vectors in Gaussian vector autoregressive models. Econometrica 59 (6): 1551-1580.

[12] MacKinnon, J.G. 1996. Numerical distribution functions for unit root and cointegration tests. Journal of Applied Econometrics 11 (6): 601-618.

[13] MacKinnon, J.G., Haug, A.A. \& Michelis, L. 1999. Numerical distribution functions of likelihood ratio tests for cointegration. Journal of Applied Econometrics 14 (5): 563-577.

[14] Newey, W.K. \& West, K.D. 1987. A simple, positive semi-definite, heteroskedasticity and autocorrelation consistent covariance matrix. Econometrica 55 (3): 703708.

[15] Ng, S. \& Perron, P. 1995. Unit root tests in ARMA models with data dependent methods for the selection of the truncation lag. Journal of the American Statistical Association 90 (429): 268-281.

[16] Osterwald-Lenum, M. 1992. A note with quantiles of the asymptotic distribution of the maximum likelihood cointegration rank test statistics. Oxford Bulletin of Economics and Statistics 54 (3): 461-472.

[17] Perron, P. 1997. Further evidence on breaking trend functions in macroeconomic variables. Journal of Econometrics 80 (2): 355-385.

[18] Perron, P. 1989. The great crash, the oil price shock, and the unit root hypothesis. Econometrica 57 (6): 1361-1401.

[19] Phillips, P.C.B. \& Perron, P. 1988. Testing for a unit root in time series regression. Biometrika 75 (2): 335-346. 